\title{
Route-Metric-Based Dynamic Routing and Wavelength Assignment for Multifiber WDM Networks
}

\author{
Jong-Seon Kim, Daniel C. Lee, Harsha Sridhar
}

\begin{abstract}
We present three dynamic routing and wavelength assignment (RWA) algorithms that are intended for multifiber WDM networks without wavelength converters. For networks with a large number of wavelengths per fiber and/or a large number of fibers per link, our algorithms exhibit particularly good performance, and one of them significantly outperforms other existing algorithms. In response to a connection request, our algorithms first select a physical route among a set of predetermined routes on the basis of some per-route variables. The algorithms then select a wavelength to be used with the selected route. This paper illustrates how we can rethink the criteria for route selection in multifiber networks. We also suggest a method of selecting the set of predetermined routes to balance the traffic load. Our simulation results illustrate that this method exhibits significantly lower blocking probability than the traditional shortest-route-based method. Thus, this paper also illustrates that the choice of predetermined routes can significantly affect the blocking probability of an RWA.
\end{abstract}

Index Terms - Multifiber WDM networks, routing, wavelength assignment

\section{INTRODUCTION}

Optical wavelength division multiplexing (WDM) technology has been receiving much attention for its potential use in a broadband network infrastructure, and the optical network technology is rapidly advancing $[1,2]$. Presently, an optical fiber can carry more than a hundred wavelengths, and this capacity will increase as the progress of dense WDM technology advances. In this paper, we present three dynamic routing and wavelength assignment (RWA) algorithms suitable for multifiber WDM networks in which each fiber can carry a large number of wavelengths. A multifiber network [3-6], in

Some part of this paper was presented at the $O F C^{\prime} 03$ Conference, March 2003.

J.-S Kim is with the Samsung Electronics Co., Ltd., Yongin, 449-901, Korea (email: jongseon.kim@samsung.com).

D. C. Lee is with the School of Engineering Science at Simon Fraser University, Burnaby, BC, Canada, V5A 1S6. (email: dchlee@ sfu.ca).

H. Sridhar is with the Department of Computer Science and Engineering at Wright State University (Sridhar.2@wright.edu).

The authors would like to thank Ohio Supercomputer Center for making their supercomputing resources available for this research. which a physical link has multiple fibers bundled, can provide an economical alternative to wavelength converters [7-11], while also increasing the network capacity. Our RWA algorithms are for a multifiber WDM network without wavelength converters, which implies that they operate under the wavelength continuity constraint [12].

For clarity, we first define a few terms. We represent the network topology by graph $(N, E)$, where $N$ represents the set of nodes (representing optical cross-connects) and $E$ represents the set of links. In a multifiber network, each link can have multiple fibers. A route is a sequence of nodes, $n_{0}, n_{1}, n_{2}, \cdots, n_{L}$, in which each adjacent pair $\left(n_{i}, n_{i+1}\right)$ is connected by a "link." This "route" can be alternatively represented by a sequence of links, $l_{1}, l_{2}, \cdots, l_{L}$, where $l_{i}$ denotes the link from node $n_{i-1}$ to node $n_{i}$. Each link, say $l_{i}$, can have multiple, say $F$, "fibers," and for our explanation we denote these fibers by $l_{i}^{1}, l_{i}^{2}, \cdots, l_{i}^{F}$. We can choose one fiber from each link along the route and construct a fiber path by connecting them at the nodes. For example, if we select a particular fiber indexed by $j_{i}$ in link $l_{i}$ for each $i$, then the sequence of fibers $l_{1}^{j_{1}}, l_{2}^{j_{2}}, \cdots, l_{L}^{j_{L}}$ is a "fiber path" in a "route" $l_{1}, l_{2}, \cdots, l_{L}$. Thus, there may be multiple "fiber paths" in a "route." Each fiber can carry different wavelengths. We assume that all the fibers in the network carry the same set, $\Omega$, of wavelengths. Let us denote these wavelengths by $w_{1}, w_{2}, \cdots, w_{W} . \quad\left(\Omega=\left\{w_{1}, w_{2}, \cdots, w_{W}\right\}\right)$ Then, each "fiber path" can simultaneously carry up to $W$ wavelengths. For example, "fiber path" $l_{1}^{j_{1}}, l_{2}^{j_{2}}, \cdots, l_{L}^{j_{L}}$ can carry wavelengths $w_{1}, w_{2}, \cdots, w_{W}$, and these different wavelengths in the same fiber path can carry different information streams. By "lightpath" [13] we refer to a particular wavelength in a particular fiber path. Thus, a "lightpath," in our discussion of multifiber networks, is identified by a combination of a "wavelength" $(w \in \Omega)$ 
and a "fiber path." To establish a connection, it is necessary to set up a lightpath in a WDM network.

We can categorize conventional dynamic RWA algorithms into two groups. In providing a lightpath in response to a connection request from a source to a destination, some algorithms -e.g., least congested path (LCP) [14], fixed-paths least-congestion (FPLC) [15], least loaded routing (LLR) [16] - select a route for the lightpath from a set of predetermined routes. If there is no available lightpath that can be established with the routes in the set of predetermined routes, the connection request is denied. The other group of algorithms consider all the available routes from a source to a destination and choose one (typically the shortest route with an appropriate distance metric $[5,6]$ ) among them. Thus, while the algorithms in the second group have more flexibility in finding a lightpath, those in the first group have less computational complexity.

In this paper, we present three predetermined-routebased dynamic RWA algorithms for multifiber WDM networks without wavelength conversion. These algorithms first select a physical route among a set of predetermined routes on the basis of the following perroute variables: the number of available lightpaths in the route and/or the current load of links along the route. (The meanings of these variables will be more clearly presented in Section II.) The algorithms then select a wavelength to be used with the selected route. With this approach, we construct an algorithm that outperforms existing algorithms [5,16] for networks with a large number of wavelengths per fiber and/or a large number of fibers per link. (Among the RWA algorithms that we referred to above, LLR [16] and SPREAD [5] have been proposed for multifiber WDM networks while the others $[6,14,15]$ have been proposed for single-fiber WDM networks.) This paper thus illustrates that in designing a "heuristic" RWA algorithm for "multifiber" networks, a separation between routing and wavelength assignment problems with an appropriate route metric to select the desired route can have positive effects on reducing the blocking probability. Many cleverly constructed existing RWA algorithms for multifiber WDM networks, while exhibiting excellent performance, end up not considering these per-route variables because such algorithms select the best individual "combination" of route and wavelength on the basis of the metric associated with each combination. For example, in response to a connection request from node $s$ to node $d$, SPREAD [5] considers all the combinations, $\left\{(w, p) \mid w \in \Omega, p \in \quad \Pi_{s d}\right\}$ of wavelength $(w)$ and route $(p)$. (We denote by $\Pi_{s d}$ the set of all routes from $s$ to $d$.) There is a particular metric that assigns a goodness value to each combination $(w, p)$, and
SPREAD selects the combination $(w, p)$ that has the best such value.

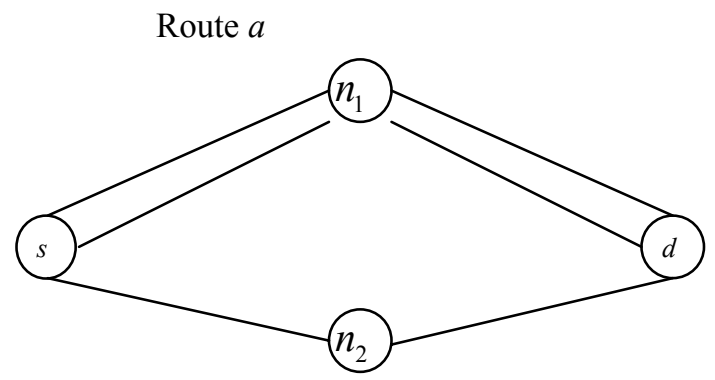

Route $b$

Fig: 1(a)

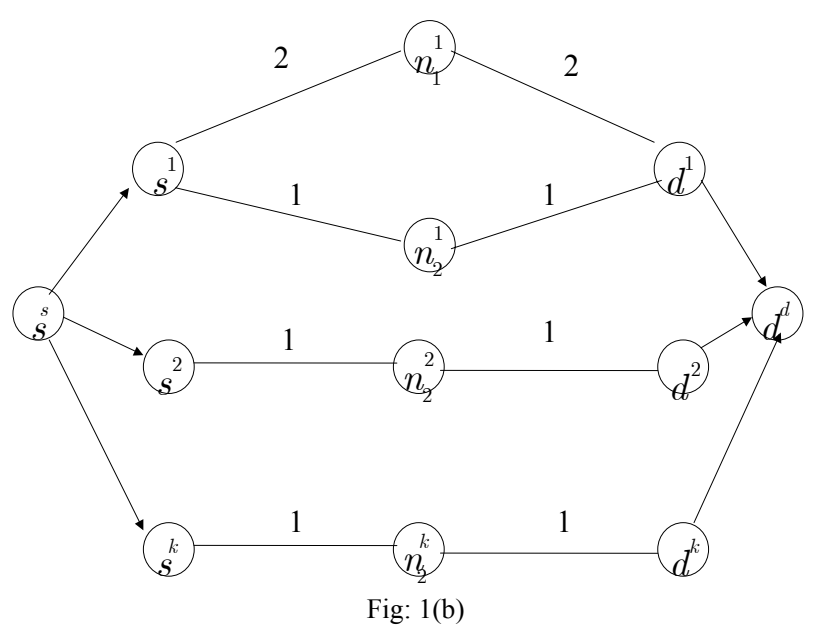

Fig. 1 (b) shows the layered graph associated with the graph in (a). In (b), the weights of links indicate the numbers of available channels (combinations of the wavelength and fiber). The maximum-weight path in this layered graph from $s^{s}$ to $d^{d}$ is through $\left(s^{1}, n_{1}^{1}, d^{1}\right)$, which corresponds to wavelength $w_{1}$ combined with route $a$ in Fig 1 (a).

To illustrate the effect of such route selections on the blocking probability, let us consider the following network status. Only two routes with the same hop length (called routes $a$ and $b$ ) are available between two nodes, and these routes are link-disjoint. Fig. 1 (a) illustrates this. Among $W$ wavelengths ( $W$ denotes the number of wavelengths per fiber), only one wavelength, $w_{1}$, is available on each link in route $a$, while $k(>2)$ wavelengths, $w_{1}, w_{2}, \cdots, w_{k}$, are available on each link in route $b$. On route $a$, each link has two fibers in which the wavelength $w_{1}$ is available. Only one fiber in each link of route $b$ is available for each of the $k$ available wavelengths. The best performing algorithms in literature for multifiber networks, SPREAD [5] and LLR [16], would consider the following combinations of wavelength and route: $\left(w_{1}, a\right),\left(w_{1}, b\right),\left(w_{2}, b\right), \cdots$, 
$\left(w_{k}, b\right)$. SPREAD [5] and LLR [16], would select route $a$ because their selection is based on a metric that is associated with individual combinations of route and wavelength. (For example, SPREAD would select combination $\left(w_{1}, a\right)$ to set up a lightpath for the connection request for the following reason. For the available wavelength $w_{1}$, each link in route $a$ has two available fibers. For a wavelength $w_{i}, 1 \leq i \leq k$, each link in route $b$ has only one fiber available. Therefore, $\left(w_{1}, a\right)$ is the winner among $\left(w_{1}, a\right), \quad\left(w_{1}, b\right)$, $\left(w_{2}, b\right), \cdots,\left(w_{k}, b\right)$ in SPREAD algorithm. Incidentally, in SPREAD the mechanism to choose a route and the mechanisms to choose a wavelength are simultaneous because the route and the wavelength are determined by the choice of the path in the "layered graph" [5], which is illustrated in Fig. 1 (b). (The detailed rule for such a choice is presented in [5] with the key concept of its "layered graph.") However, choosing route $a$ may not be good for reducing the blocking probability because links in route $a$ have fewer channels (wavelength-fiber combinations) available for future connection requests from other node pairs. (In this example, each link in route $a$ has two available channels. Let us denote the two available fibers for wavelength $w_{1}$ in each link in route $a$ by $f_{1}$ and $f_{2}$. Then, the two channels are $\left(w_{1}, f_{1}\right)$ and $\left(w_{1}, f_{2}\right)$. Each link in route $b$ has $k$ available channels. Let us denote by $f_{1}$ the only available fiber for wavelengths $w_{1}, w_{2}, \cdots, w_{k}$ in each link in route $b$. Then, each link in route $b$ has available channels $\left(w_{1}, f_{1}\right)$, $\left(w_{2}, f_{1}\right), \cdots,\left(w_{k}, f_{1}\right)$.) Based on this kind of reasoning, our algorithms would select route $b$. This reasoning of favoring route $b$ instead of route $a$ is even more appealing for a larger value of $k$. Thus, we can speculate that our algorithms' performance relative to SPREAD [5] or LLR [16] will be better for a network with a larger number of wavelengths.

Our algorithms were motivated by the intuition that in selecting a route one should consider the number of available wavelength-fiber combinations in the links constituting each candidate route. Based on this intuition, we designed a metric to be used as the route-selecting criterion that is associated with each route rather than each route-wavelength combination. Our algorithm selects the wavelength in accordance with a wavelength selection rule after the route has been selected. Simulations show that our dynamic RWA algorithms exhibit better performance than existing algorithms for networks with a large number of wavelengths per fiber and a large number of fibers per link. Thus, the results in this paper will establish that for a multifiber network, routing decisions based on such perroute variables can have positive effects on performance for networks with a large number of wavelengths per fiber and/or a large number of fibers per link.

Our algorithms use a set of predetermined routes, and the off-line selection of this set affects the blocking probability. In this paper, we also present predetermined route-selection methods that balance the load in the network in accordance with the traffic intensities of different source-destination $(s-d)$ pairs. In past studies, a set of predetermined routes for each $s-d$ pair was typically selected by progressively choosing minimum-hop routes so that the routes in the set were non-overlapping. For example, if two predetermined routes are to be selected for an $s-d$ pair, the minimum-hop route is first selected, and the second route is added by selecting the route that has minimum hops among all the routes link-disjoint with the first route. However, this method may cause congestion in some links while other links are underutilized because the predetermined routes are independently chosen for each $s$ $d$ pair without considering the predetermined routes for other $s-d$ pairs. In contrast, we formulate the predetermined route selection problems as integer linear programming (ILP) problems to balance loads, with the ultimate goal in mind of reducing the blocking probability. Simulation results presented in section IV will show that selecting predetermined routes for a balanced load significantly reduces the blocking probability even further.

The rest of the paper is organized as follows. In Section II, we present our route metric and RWA algorithms. Section III proposes the problem formulations for predetermined route selection by means of ILP. In Section IV, our proposed algorithms are evaluated and compared with other existing algorithms through simulations. Section V contains our conclusion.

\section{ROUTING AND WAVELENGTH ASSIGNMENT ALGORITHMS}

In this section we present three routing and wavelength assignment algorithms. Each of these algorithms selects, on the basis of its own per-route metric, a route from a predetermined route set. Let $U_{i j}{ }^{w}$ be the number of unoccupied fibers for wavelength $w$ on link $i j$. Note that we are considering multifiber networks. We denote by $W_{p}$ the set of available wavelengths for the route $p$ at the time of connection request arrival. (Note that $W_{p}$ dynamically changes with the state of the network.)

\section{A. Maximum-Channel Routing (MCR)}

In this paper, the number of available channels in a route refers to the number of lightpaths that can be established simultaneously in the route at a time. The bottleneck link for wavelength $w$ in a route is the link that has the minimum number of $U_{i j}{ }^{w}$ for the route. The number of available channels for a wavelength in a route is defined as the number of unoccupied fibers in the bottleneck link of the route for the wavelength. Therefore, the bottleneck link for each wavelength may or may not be the same. For 
each route, the number of available channels for all wavelengths is summed. The number of available channels in route $p$ can be expressed as:

$$
\sum_{w \in W_{p}} \min _{i j \in p} U_{i j}^{w} .
$$

(In the same route, if one wavelength can be carried in different fibers in each link along the route, the same wavelength in the same route can yield multiple lightpaths.)

The routing and wavelength assignment scheme for the MCR algorithm is as described below. Upon the arrival of each connection request, the MCR algorithm selects the route $p$ that attains

$$
\max _{p}\left\{\sum_{w \in W_{p}} \min _{i j \in p} U_{i j}^{w}\right\} .
$$

Thus, the MCR algorithm selects the least congested route among the predetermined routes by considering the number of available channels. When multiple routes have the same number of available channels, the route with the minimum hop count among them will be chosen. On the selected route $p_{s}$, the MCR algorithm then chooses the wavelength $w$ that attains

$$
\max _{w}\left\{\min _{i j \in p_{s}} U_{i j}^{w}\right\} \text {. }
$$

The wavelength with minimum congestion is assigned from the available wavelengths on the selected route $p_{s}$. If there is a tie for the maximum number of available channels in multiple wavelengths, we use the first-fit wavelength assignment scheme [12] to choose a wavelength. First-fit scheme performs well in terms of blocking probability and is preferred in practice because of its small computational overhead and low complexity. We note that the route selection part of MCR can be reduced to that of FPLC [15] in a single-fiber system.

We now briefly discuss computational complexity of the on-line processing of MCR algorithm - that is, the computation required to select a route based on criterion (2) and to select a wavelength based on criterion (3). We denote by $W$ the number of wavelengths in each fiber. We denote by $l(p)$ the number of links in route $p$. We denote by $Q_{s d}$ the set of predetermined routes from source $s$ to destination $d$, and by $q_{s d}$ we denote the number of predetermined routes from source $s$ to destination $d$ ( $q_{s d}=\left|Q_{s d}\right|$ ). In response to a connection request from $s$ to $d$, selecting a route in accordance with (2) has computational complexity

$$
O\left(\sum_{p \in Q_{s d}} W l(p)\right)=O\left(W \sum_{p \in Q_{s d}} l(p)\right) .
$$

After performing computation for selecting a route, say $p$, additional computation necessary to select to a wavelength in accordance with (3) is only $\left|W_{p}\right|$ comparisons. Thus, we can express this additional computational complexity as $O(W)$, and the complexity of the total call processing is $O\left(W \sum_{p \in Q_{s d}} l(p)\right)$.

\section{B. Least-Sum-Normalized-Load Routing (LSNLR)}

In the LSNLR algorithm, the $\operatorname{cost} C_{i j}{ }^{w}$ for wavelength $w$ on link $i j$ is defined as follows:

$$
C_{i j}^{w}=1-\frac{U_{i j}^{w}}{F_{i j}},
$$

where $F_{i j}$ is the number of fibers on link $i j$. (Note, first, that this cost can be interpreted as the utilization of the link for wavelength $w$, and, second, that high utilization of the link can be seen as creating a highly loaded link.) As a route-selection criterion, the LSNLR algorithm uses the following metric:

$$
\sum_{w \in W_{p}} \sum_{i j \in p} C_{i j}^{w} /\left|W_{p}\right|^{2}=\frac{\sum_{w \in W_{p}} \sum_{i j \in p} C_{i j}^{w}}{\left|W_{p}\right|} \frac{1}{\left|W_{p}\right|} .
$$

-e.g., choosing the route with the minimum value of (5). Expression $\sum_{i j \in p} C_{i j}^{w}$ can be viewed as the cost associated with wavelength $w$ and route $p$.

Then, expression

$$
\left(\sum_{w \in W_{p}} \sum_{i j \in p} C_{i j}^{w}\right) /\left|W_{p}\right|
$$

can be interpreted as the cost of route $p$ per available wavelength. The factor $1 /\left|W_{p}\right|$ in (5) gives preference to the route that has more wavelengths available. (We empirically found that this factor significantly improves performance.) In short, upon the arrival of each connection request, the LSNLR algorithm selects the route $p$ that achieves

$$
\min _{p}\left\{\left(\sum_{w \in W_{p}} \sum_{i j \in p} C_{i j}^{w}\right) /\left|W_{p}\right|^{2}\right\} .
$$

The LSNLR algorithm then chooses the wavelength $w$ on the selected route $p_{s}$ that in accordance with criterion (3). (We also tried the following criteria:

$$
\min _{w}\left(\sum_{i j \in p_{s}} C_{i j}^{w}\right)
$$

-that is, to choose the wavelength with minimum sum of load. We empirically found that criteria (3) yields slightly better performance.) The tie-breaking rules for routing and wavelength assignment are the same as in the MCR algorithm. 
Computational complexity for selecting a route in accordance with (5) is $O\left(W \sum_{p \in Q_{s d}} l(p)\right)$. After performing the computation for selecting a route, selection of the wavelength in accordance (3) or (8) takes only additional $\left|W_{p}\right|$ comparisons. Thus, computational complexity for call processing is $O\left(W \sum_{p \in Q_{s d}} l(p)\right)$.

At this point, we briefly compare MCR and LSNLR. From the above discussions we can see that they both have the same computational complexity. We acknowledge that both algorithms are developed on the basis of intuition and are empirically evaluated, as most of dynamic routing and wavelength assignment algorithms are. While the main concern of MCR is to protect a bottleneck link, the conceptual underpinning of LSNLR is to maximize the total availability of communication channels in the network.

\section{C. $F(w, l)$}

Both MCR and LSNLR use route metrics that are intuitively appealing. However, we can run into the following network status: one route is much more attractive than another in terms of the LSNLR metric (7) while they are more or less equally attractive in terms of the MCR metric (1), or, alternatively, one route is much more attractive than another in terms of the MCR metric (1) while they are more or less equally attractive in terms of the LSNLR metric (7). Therefore, in this section we suggest a route metric that combines (1) and (7).

The routing and wavelength assignment scheme for the proposed algorithm can be formulated as follows. Upon the arrival of each connection request, the algorithm selects the route $p$ that achieves

$$
\min _{p}\left\{\frac{\sum_{w \in W_{p}} \sum_{i j \in p} C_{i j}^{w}}{\left|W_{p}\right|^{2} \sum_{w \in W_{p}} \min _{i j \in p} U_{i j}^{w}}\right\} .
$$

The route metric used in (9) combines the metrics used in (1) and (7). If a route has a favorable value of both metrics (1) and (7), metric (9) will have a very favorable value. If a route has a predominantly favorable value of metric (1) while having a mediocre value of (7), or if a route has a predominantly favorable value of metric (7) while having a mediocre value of (1), then metric (9) will have a favorable value. We note here that the metric used in (9) is designed based on our trials with many other designs of the route metric. Let us consider the network status that determines the value of the metric in (9). We denote by $F(w, l)$ the number of link $l$ 's available fibers in which wavelength $w$ is available. The value of the metric in (9) for route $p$ is determined by $\{F(w, l) \mid \forall l \in p, \forall w\}$.
For lack of a better name, we call the metric used in (9) the $F(w, l)$ metric. When multiple routes have the same value of $F(w, l)$ metric, the route with the minimum hop count is chosen. As for the selection wavelength $w$ on the selected route $p_{s}$, either criterion (3) or (8) can be used. We found that the wavelength assignment scheme (3) slightly outperforms (8) in most of our simulations. Therefore, we suggest using (3) for wavelength selection of $F(w, l)$.

Computational complexity of $F(w, l)$ scheme for processing a call request is $O\left(W \sum_{p \in Q_{s d}} l(p)\right)$ for the call from source $s$ to destination $d$. If the number of predetermined routes are fixed, which is typical in applying the proposed algorithms, then the complexity can be conservatively expressed as $O(W N)$, where $N$ is the number of nodes in the physical network. This is relatively low. For example, SPREAD has complexity $O(\max \{N, W\} \times W N)$.

In Fig. 3, the blocking probabilities of the proposed algorithms, MCR, LSNLR, and F(w,l), and the well known algorithms, LLR, SPREAD, are presented and compared for the network topology illustrated in Fig. 2 (a). The traditional shortest-route-based method was used to select a set of predetermined routes for LLR, MCR, LSNLR, and $\mathrm{F}(\mathrm{w}, \mathrm{l})$, and their performance curves are denoted by LLRSP, MSP, LSP, and F(w,l)SP, respectively. (Section IV presents more details of simulations.) Fig. 3 shows that for networks with a large number of wavelengths per fiber and/or a large number of fibers per link, $F(\mathrm{w}, \mathrm{l})$ exhibits a blocking probability superior to existing algorithms (LLR and SPREAD). 


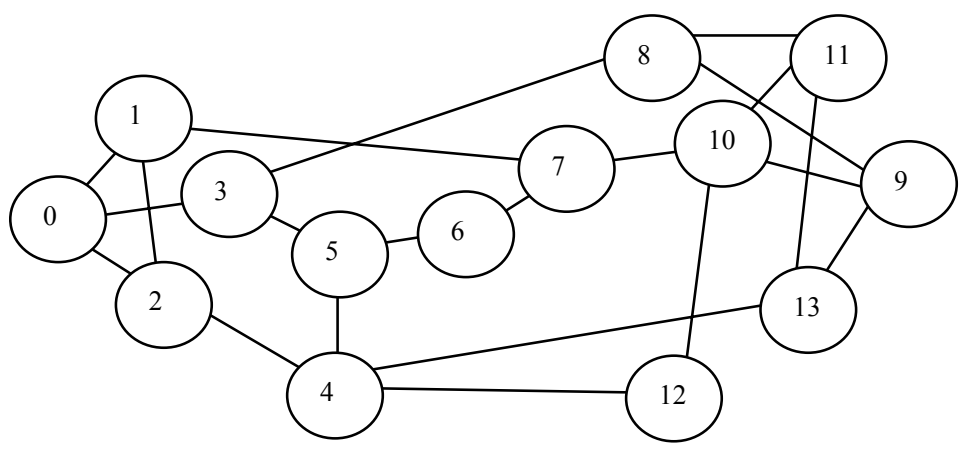

a) NSFNET

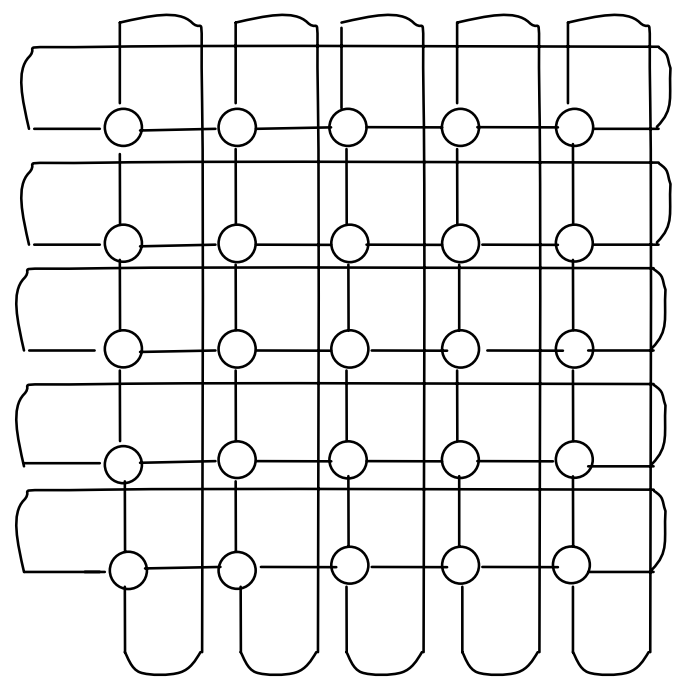

b) $5 \times 5$ mesh torus

Fig. 2. Network topologies used in the simulations. 


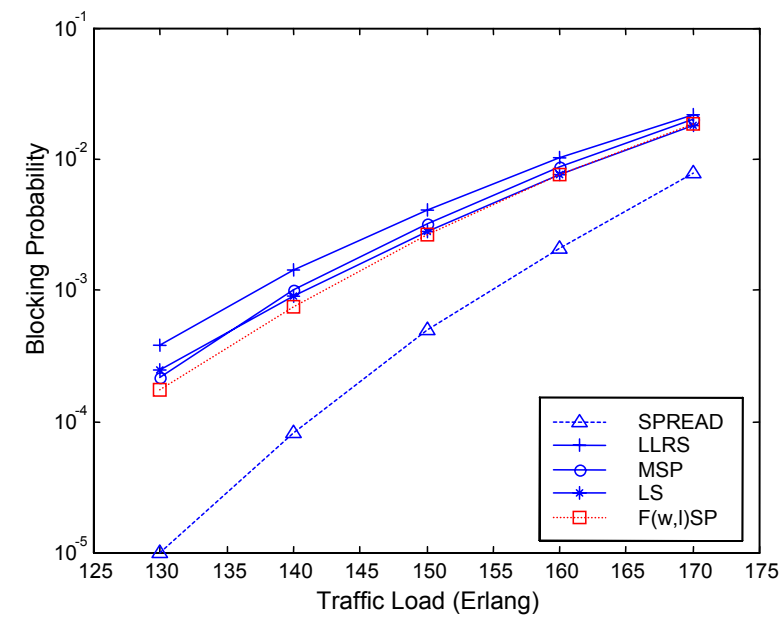

a) $\operatorname{NSFNET}(W=4, F=4)$
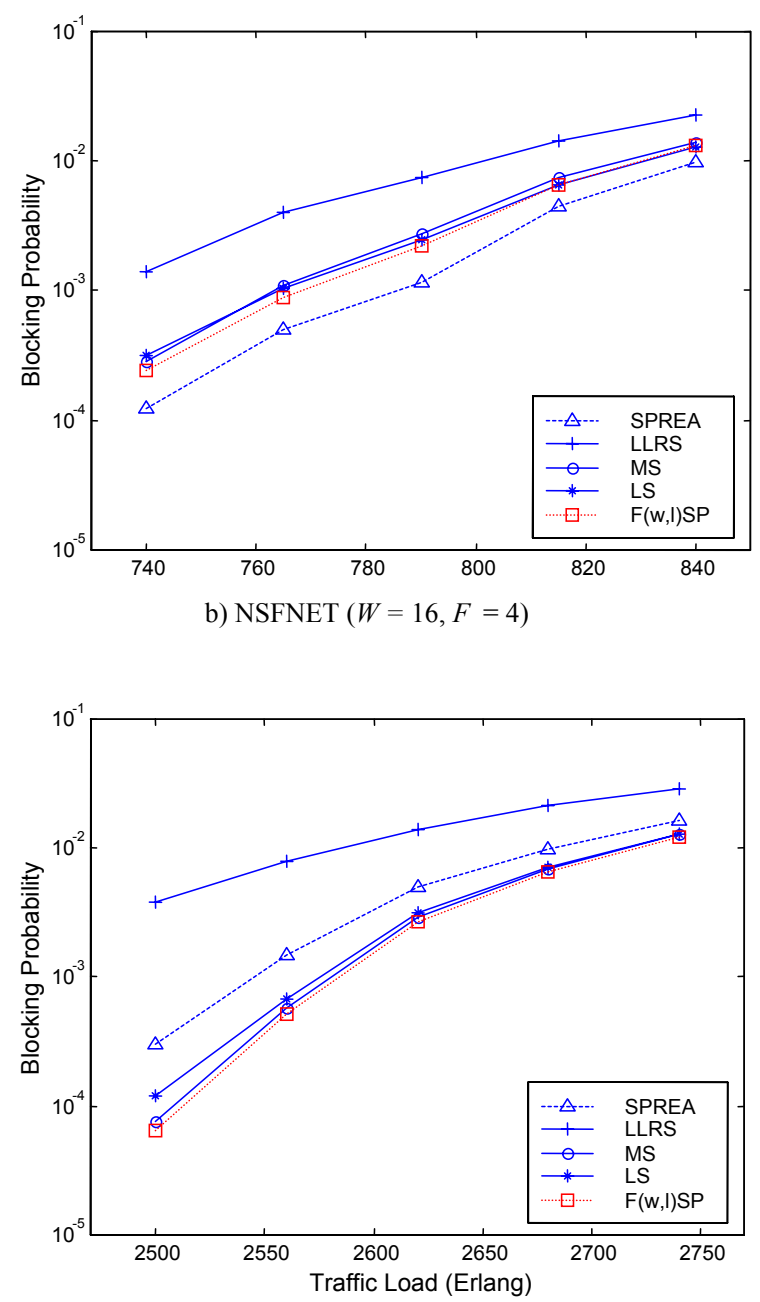

d) NSFNET $(W=48, F=4)$

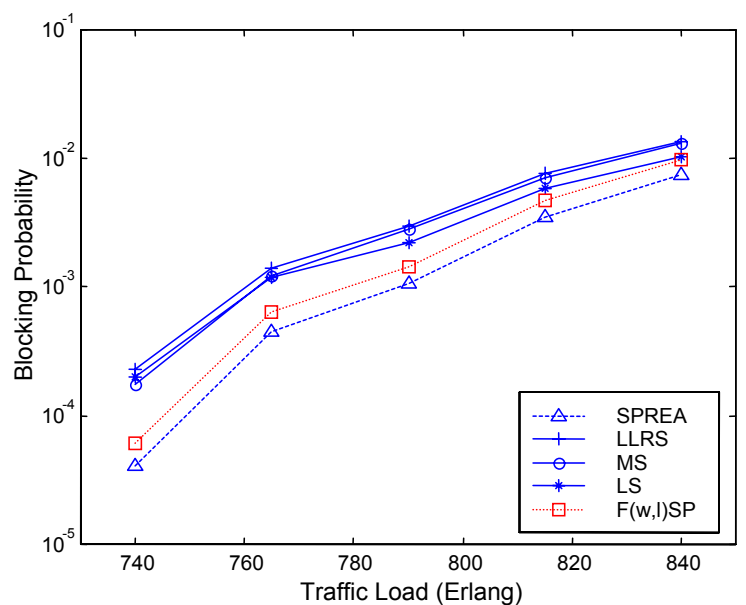

c) NSFNET ( $W=4, F=16$ )

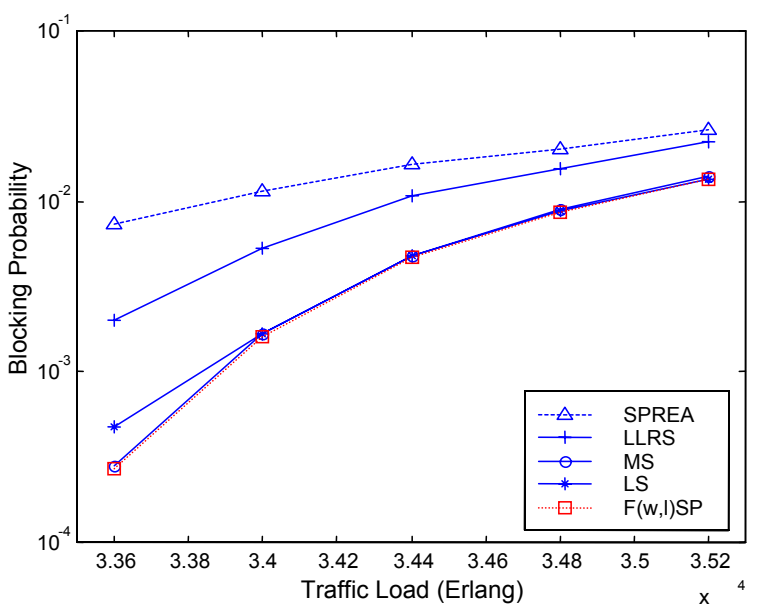

e) $\operatorname{NSFNET}(W=48, F=48)$

Fig 3) Blocking probability vs traffic load in NSFNET using shortest-path-based route selection (uniform traffic). 


\section{OfF-Line Selection of PREDETERMined RouteS}

In existing RWA algorithms that employ a set of predetermined routes, the set for each $s$ - $d$ pair is typically selected by progressively choosing minimum-hop routes so that the routes in the set are link-disjoint. However, this shortest-route-based selection may cause congestion in some links while other links are underutilized because the predetermined routes are independently chosen for each $s$ $d$ pair without considering the predetermined routes for other $s-d$ pairs. We note that the selection of predetermined routes should be done with a global perspective in order to achieve a low blocking probability. We also note that the selection should take into consideration different traffic intensities (arrival rates of connection requests) of different $s-d$ pairs. (We consider non-uniform traffic as well as uniform traffic.) Based on these considerations, in this section we formulate the predetermined route selection problem as optimization (integer linear programming) problems with objective functions specifically designed so that the optimal solutions (i.e., the optimal set of predetermined routes) result in low blocking probability. Simulation results in Section IV show that the selection of predetermined routes through these optimizations, results in significantly lower blocking probabilities than the traditional shortest-routebased selection.

The optimization problems are constructed based on the network topology, traffic intensity matrix, and the number of predetermined routes for each $s$ - $d$ pair (sourcedestination pair), and solutions to the problems represent sets of predetermined routes. Much like in the traditional method, in our proposed formulations link-disjoint predetermined routes are selected for each $s-d$ pair. We use the following notations:

- $s$ and $d$ : source and destination nodes, respectively,

- $\quad i j$ : link between node $i$ and node $j$,

- $q_{s d}$ : number of predetermined routes from source $s$ to destination $d$,

- $n$ : indexing of predetermined routes for an $s$ - $d$ pair, $n=1,2, \ldots, q_{s d}$,

- $\quad c_{i j}$ : existence of a link in the physical topology $\left(c_{i j}\right.$ $=1$ if there is a physical link from node $i$ to node $j, c_{i j}=0$ otherwise),

- $\quad \lambda_{s d}$ : traffic intensity for the $s$ - $d$ pair (arrival rate of the requests for connection from $s$ to $d$ )

- $x_{i j}^{s d n}: 1$ if there is the $n^{\text {th }}$ predetermined route from $s$ to $d$ passing through the link $i j$, and 0 otherwise (This is the decision variable for predetermined route selection).

We define the load on link $i j$ as

$$
\frac{\sum_{s, d, n} x_{i j}^{s d n} \lambda_{s d}}{F_{i j}},
$$

where $F_{i j}$ is the number of fibers in link $i j$. We define the total link usage as the sum of the load on each link in the network. If a link has the maximal load among all the links in the network, we refer to this link as the most congested link. Given the network topology, the traffic intensity matrix, and the number of predetermined routes for each $s$ $d$ pair, we consider the following two formulations.

- Formulation 1 (F1): Minimize the total link usage under minimal loading on the most congested link.

- Formulation 2 (F2): Minimize the load on the most congested link under minimal total link usage.

Each problem formulation consists of two ILPs, one of which is used to provide a constraint to the other ILP.

Formulation 1:

F1-a: Mixed Integer Programming

Objective: Minimize $x_{\max }$

Subject to:

$x_{\max } \geq \frac{\sum_{s, d, n}\left(x_{i j}^{s d n} \lambda_{s d}\right)}{F_{i j}} \forall i, j$
$\sum_{i, n} x_{i s}^{s d n}-\sum_{k, n} x_{s k}^{s d n}=-q_{s d} \quad \forall s, d$

$\sum_{i, n} x_{i d}^{s d n}-\sum_{k, n} x_{d k}^{s d n}=q_{s d} \quad \forall s, d$

$\sum_{i} x_{i j}^{s d n}-\sum_{k} x_{j k}^{s d n}=0 \quad \forall j, s, d, n(j \neq s$ and $j \neq d)$

$\sum_{n} x_{i j}^{s d n} \leq c_{i j} \quad \forall s, d, i, j$

$\sum_{i} x_{s i}^{s d n}=1, \quad \forall s, d, n$

$\sum_{i j} x_{i j}^{s d n} \leq \sum_{i j} x_{i j}^{s d(n+1)} n=1,2, . ., q_{s d}-1, \quad \forall s, d$,

$x_{i j}^{s d n} \in\{0,1\}$.

In order to use Linear Programming formulation and available tools, we have transformed a minmax problem into a minimization problem using equations (10) and (11). Expressions (10) and (11) show that the objective of this 
formulation is to minimize the load on the most congested link. Inequality (11) indicates that in balancing the loads of links, we are considering different numbers of fibers in different links and also different traffic intensities of different source-destination pairs. Equations (12), (13), and (14) ensure that the given number of predetermined routes for each $s$ - $d$ pair exists. At each source node $s$, equations (12) and (13) impose the constraint that the number of predetermined routes from $s$ to $d$ is equal to

$q_{s d}$. At each intermediate node $j$, equation (14) shows that the incoming predetermined route index for each $s-d$ pair should be the same as the outgoing predetermined route index for the same $s$ - $d$ pair. Equation (15) ensures that a predetermined route on an $s-d$ pair should be linkdisjoint to all other predetermined routes for the $s-d$ pair. It also ensures that the predetermined route passes through a link on the physical topology. Equation (16) guarantees that only one route index is assigned to each predetermined route. In place of (16), we could use constraint, $\sum_{i} x_{i d}^{s d n}=1, \forall s, d, n$. The purpose of constraint (17) is to index the predetermined routes of each source-destination pair in the increasing order of their number of hops. (Inequality (17) is non-essential. If the size of the linear programming is computationally burdensome, inequality (17) can be omitted.) This route index information can be used in solving the route selection problem. For example, if the tie-breaking rule that chooses the route over which the smaller number of hops is used, we can simply select the route that has the smallest predetermined route index without counting the number of hops again. Equation (18) represents the integral constraint for the decision variable. This decision variable should consist of 0 or 1 .

Note that the number of variables grows as $O\left(L \sum_{s, d} q_{s d}\right)$, where $L$ denotes the number of links in the physical network, and that the number of constraints grows as $O\left(N \sum_{s, d} q_{s d}\right)$, where $N$ denotes the number of nodes in the network. The optimal (minimal) load on the most congested link is used in F1-b ILP as a constraint and is referred to as $x_{\text {opt. }}$.

\section{$\underline{\text { F1-b ILP }}$}

Objective: $\quad$ Minimize $\sum_{i, j, s, d, n} \frac{x_{i j}^{s d n} \lambda_{s d}}{F_{i j}}$
Subject to:

$$
x_{\mathrm{opt}} \geq \frac{\sum_{s, d, n}\left(x_{i j}^{s d n} \lambda_{s d}\right)}{F_{i j}} \forall i, j
$$

and constraints (12)-(18).

Equation (19) shows the objective function that minimizes the total link usage with consideration of the traffic intensity from any $s$ to any $d$. Equation (20) ensures that the load on each link is not greater than the optimal value (the minimized load on the most congested link) provided by F1-a ILP. The predetermined routes for each $s-d$ pair extracted from the decision variables $\left(x_{i j}^{s d n}\right)$ will be used in our simulations as one of the predetermined routeselection methods.

Formulation 2:

$\underline{\text { F2-a ILP }}$

Objective function: Minimize $\sum_{i, j, s, d, n} \frac{x_{i j}^{s d n} \lambda_{s d}}{F_{i j}}$

Subject to: Constraints (12)-(18).

The only difference between F1-b ILP and F2-a ILP is that there is no constraint for the minimized load on the most congested link in F2-a ILP. F2-a ILP provides the minimized total link usage that becomes a constraint to F2b ILP. We refer to this optimal value as $S^{\text {opt }}$.

\section{F2-b: Mixed Integer Programming}

Objective Function: Minimize $\quad x_{\max }$

Subject to: $\quad \sum_{i, j, s, d, n} \frac{x_{i j}^{s d n} \lambda_{s d}}{F_{i j}}=S^{\mathrm{opt}}$

and constraints (11)-(18).

Equation (23) ensures that the total link usage is the optimal value, the minimized total link usage provided by F2-a ILP. The predetermined routes for each $s$ - $d$ pair extracted from the decision variables $\left(x_{i j}^{s d n}\right)$ will be used in our simulations as another predetermined routeselection method.

Figs. 4 and 5 show that the predetermined paths selection methods presented in this section can indeed achieve better blocking performance than the ones selected by the traditional shortest-route-based method. In Fig. 4, comparisons are made between the different route 
selection methods for our algorithms, MCR, LSNLR, and $\mathrm{F}(\mathrm{w}, \mathrm{l})$ (proposed in section II). Fig 5 has the same comparisons for the LLR algorithm. Figs. 4 and 5 show the blocking probabilities with three predetermined routeselection methods-i.e., ILP formulation 1, ILP formulation 2, and the traditional shortest-route-based method, which are denoted by F1, F2, and SP, respectively. More details on these simulation results are presented in section IV.

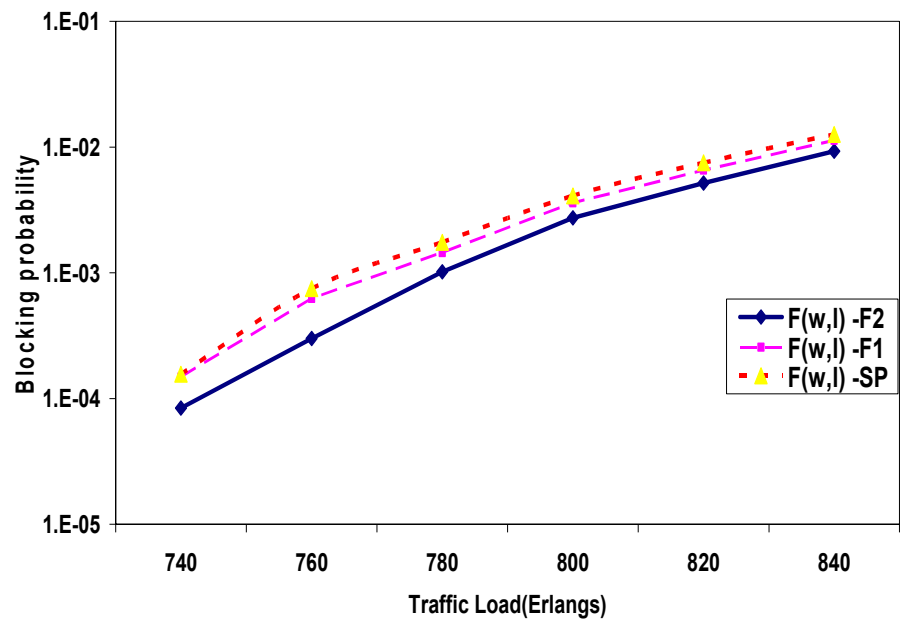

(a)

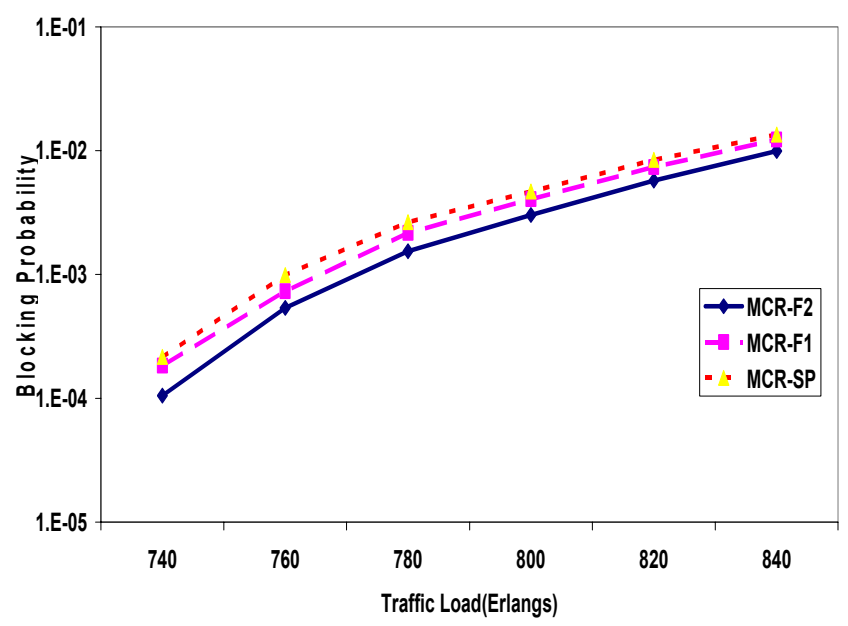

(b)

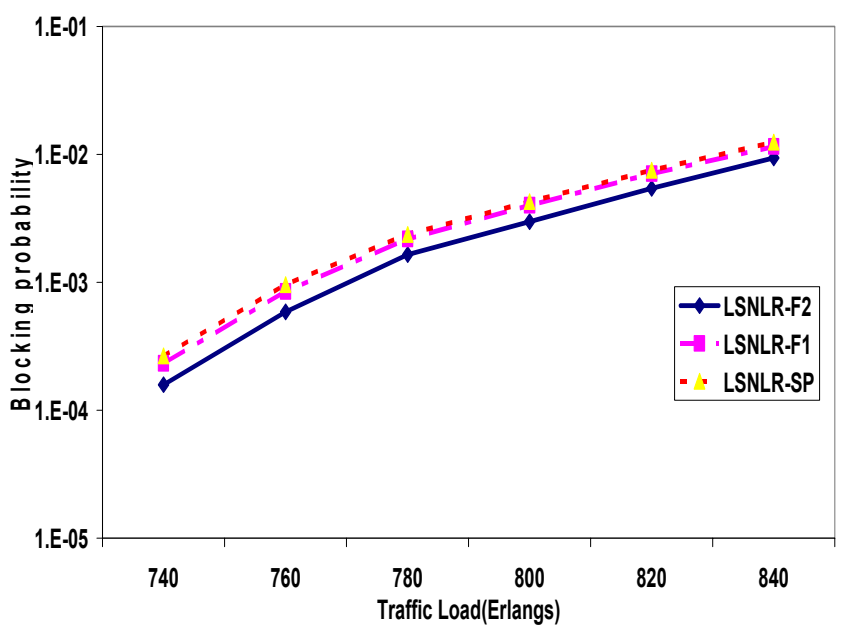

(c)

Fig 4) Blocking probability vs. traffic load in NSFNET (uniform traffic $W=16, F=4$ ) 


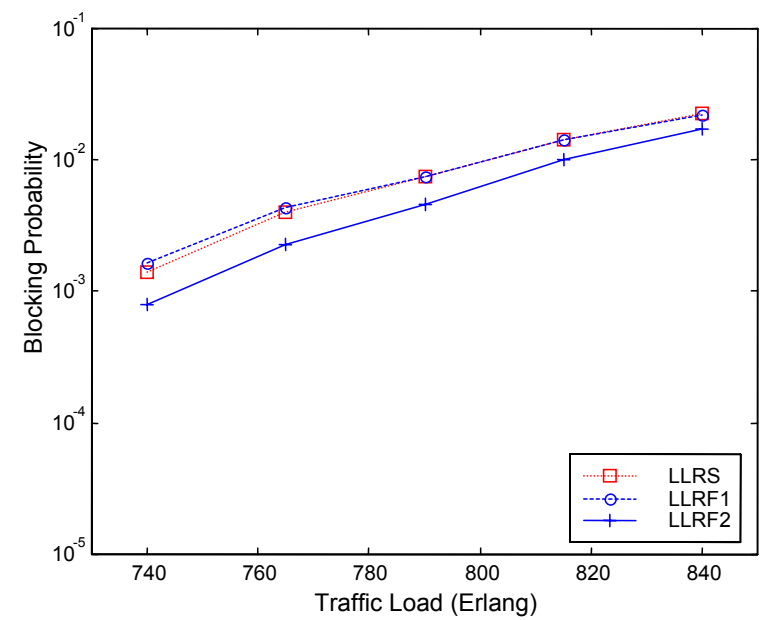

a) $W=16$ and $F=4$ (uniform traffic distribution)

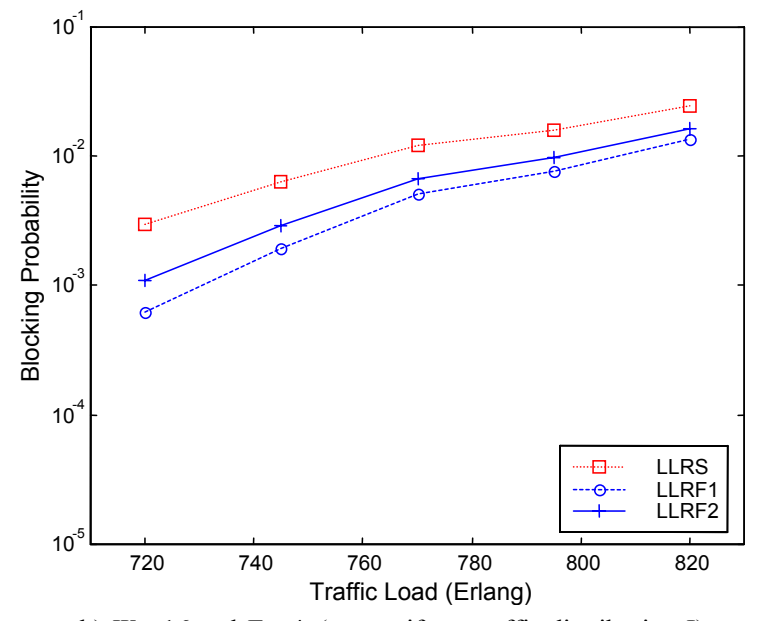

b) $W=16$ and $F=4$ (non-uniform traffic distribution-I)

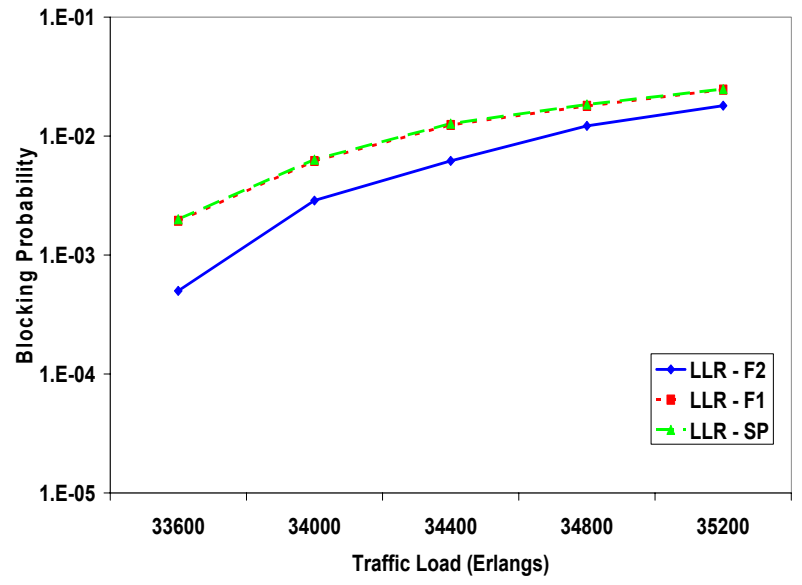

(d) $\mathrm{W}=48, \mathrm{~F}=48$ (uniform traffic)

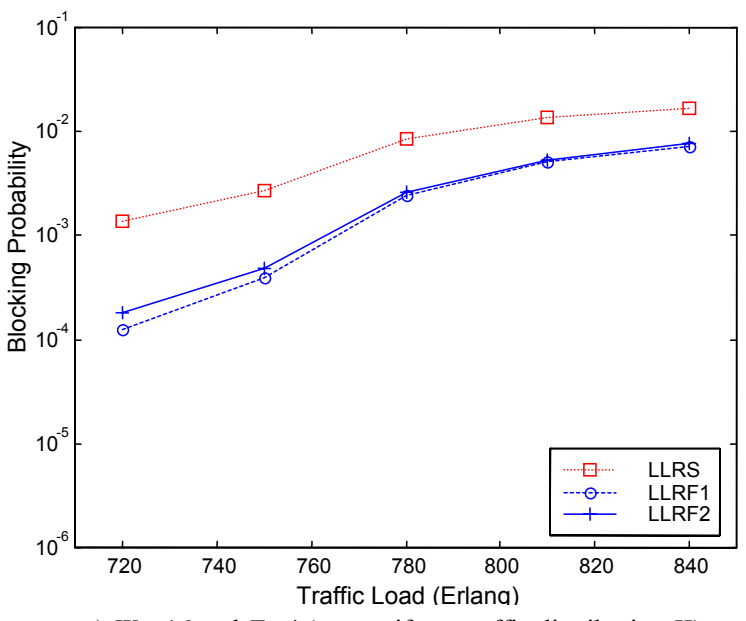

c) $W=16$ and $F=4$ (non-uniform traffic distribution-II)

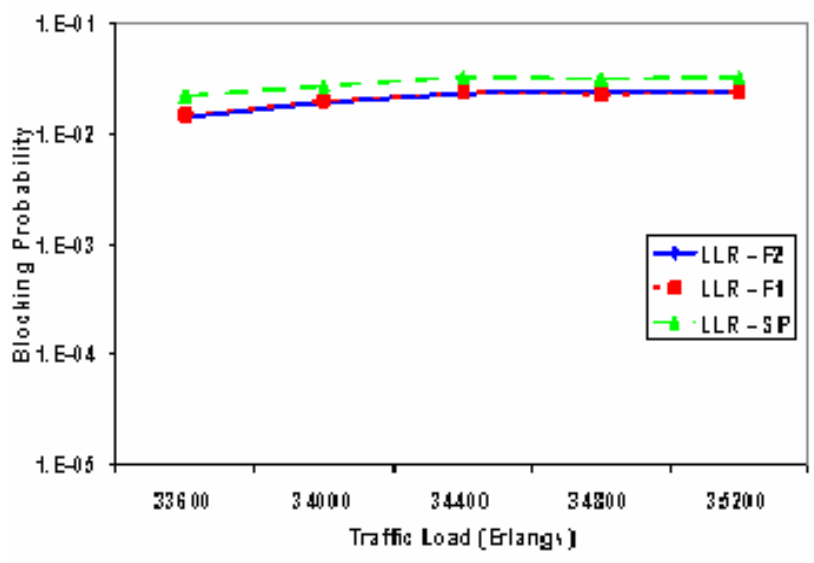

(e) W=48, F=48 (non-uniform traffic distribution-I)

Fig 5: LLR blocking probabilities in NSFNET. Non-uniform traffic distribution-I has one $s-d$ pair with heavy traffic. Nonuniform traffic distribution-II has two $s$ - $d$ pairs with heavy traffic. 


\section{SiMULATION RESULTS}

We evaluated the blocking probabilities of the proposed dynamic RWA algorithms on two network topologies. We employed the NSFNET network with 14 nodes and 21 links, to represent an irregular topology, and the $5 \times 5$ mesh torus network with 25 nodes and 50 links, to represent regular topologies for our simulations, which are illustrated in Fig. 2. For the performance comparison with existing algorithms in multifiber networks, we used LLR [16] and SPREAD [5], primarily for two reasons. First, these algorithms have outstanding performance for multifiber networks. Second, they compare individual route-wavelength combinations and select one combination on the basis of metrics associated with the route-wavelength combinations, in contrast to considering individual routes on the basis of a per-route metric.

The following configurations were used in our simulations. (1) There were an equal number of fibers per link and an equal number of wavelengths per fiber used in most of the cases. However in order to demonstrate the efficiency of the algorithm in heterogeneous environments, for some simulations we have unequal number of fibers per link. (2) For each $s$ - $d$ pair in the physical topology, in most simulations two predetermined routes (which are link-disjoint) were used for our proposed RWA algorithms. (While reading this section, readers can assume two predetermined routes unless the number of predetermined routes was stated.) (3) Connection requests for each $s$ - $d$ pair were assumed to arrive at the network in accordance with a Poisson process. The connection holding time was exponentially distributed with unit mean. Therefore, the traffic load $(\rho)$ arriving at the network was given in Erlangs as $\rho=\beta$, where $\beta$ is the total arrival rate. (4) Each set of data in our simulations was obtained from $10^{6}$ call arrivals.

The call-blocking probabilities are plotted in Figures 3-9 as a function of the traffic load arriving at the network. In Figures 3-9, $W$ and $F$ denote the number of wavelengths per fiber and the number of fibers per link, respectively. In Fig. 3, the blocking probabilities of LLR, SPREAD, MCR, LSNLR, and $\mathrm{F}(\mathrm{w}, \mathrm{l})$ are presented under the configuration that the traditional shortest-route-based method was used to select a set of predetermined routes for LLR, MCR, LSNLR, and F(w,l). (SPREAD does not use predetermined routes.) Their performance curves are denoted by LLRSP, MSP, LSP, and F(w,l)SP, respectively. Equal Poisson traffic intensity for all $s$ - $d$ pairs was assumed. Fig. 3 shows that for networks with a large number of wavelengths per fiber and a large number of fibers per link, $\mathrm{F}(\mathrm{w}, \mathrm{l})$ exhibits a blocking probability superior to existing algorithms (LLR and SPREAD).
For the simulations in Figure 3, we employed NSFNET. Figure 3 (a), (b), and (d) together show how the blocking performance of our algorithms relatively improves as the number of wavelengths per fiber increases at a fixed number of fibers. In comparison with SPREAD, at $W=4$ and $F=4$ our algorithms are much inferior. However, the performance of our algorithms is closer to that of SPREAD at $W=16$ and $F=4$. Finally, our algorithms significantly outperform SPREAD at $W=48$ and $F=4$. $\mathrm{F}(\mathrm{w}, 1)$ slightly outperforms MCR and LSNLR, and all of our proposed algorithms show better performance than LLR. The performance gap between our proposed algorithms and LLR becomes wider as $W$ grows. Figure 3 (a) and (c) show how the blocking performance of our algorithms relatively improves as the number of fibers per link increases, with the number of wavelengths being fixed. Figure 3 (e) shows the blocking performance for a large number of both wavelengths and fibers. With $W=48$ and $F=48$, our proposed algorithms significantly outperform SPREAD and LLR for NSFNET. The performance gap at $W=48$ and $F=48$ between our algorithms and SPREAD (or LLR) becomes remarkable. (Incidentally, the simulation results in Fig. 3 indicate that SPREAD performs better than LLR for small $W F$ values and worse than LLR for large $W F$ values.) In summary of Fig 3, we note that for networks with small $W F$, SPREAD performs better than our algorithms proposed in section II. However, the simulation results indicate that the comparative performance of our algorithms improves as $W F$ increases and finally becomes better than SPREAD.

Fig. 4 shows that for $F(w, 1), M C R$, and LSNLR, which were presented in section II, the offline route selection methods presented in section III make a difference in blocking performance. The curves denoted by MCR-SP, LSNLR-SP, and F(w,1)-SP show the performance of the traditional shortest-route-based method. Fig. 4 shows that in each of the three RWA algorithms, F2 formulation performs the best. The Equal Poisson traffic intensity for all $s$ - $d$ pairs was assumed.

Fig. 5 illustrates that our offline path selection method is not only good for our own RWA algorithms but also for other RWA algorithms employing predetermined routes. Fig. 5 compares the blocking probabilities of LLR schemes that employ different predetermined routeselection methods in NSFNET. Figs 5 (a), (b), and (c) are for $W=16$ and $F=4$, and Figs. 5 (d) and (e) are for $W=F=48$. LLR with our predetermined selection methods F1/F2 exhibits performance improvement in most cases. (Note, however, that in our simulations $\mathrm{F}(\mathrm{w}, \mathrm{l})$ always significantly outperformed LLR when they both use the same predetermined route-selection method - F1, F2, or SP.) In the case of LLR, F1 show better performance than $\mathrm{F} 2$ in some configurations, and F2 performs better than F1 
in most of the other configurations. Fig. 5 (a) shows the results in uniform traffic distribution while Figure 5 (b) and (c) show the results in two cases of non-uniform traffic. For non-uniform traffic in Figure 5 (b), we simply chose one $s-d$ pair and set its traffic intensity 10 times as high as other $s$ - $d$ pairs. Nodes 1 and 2 in the NSFNET topology illustrated in Figure 2 (a) were used as the $s-d$ pair that is heavily loaded. In Figure 5 (c), we set one more heavily loaded $s-d$ pair-nodes 3 and 4 in Figure 2 (a) were added as the $s$ - $d$ pair whose traffic intensity is 10 times as high as most other $s-d$ pairs. Simulations show that $\mathrm{F}(\mathrm{w}, \mathrm{l})$ in combination with our predetermined routeselection methods (denoted by F1 and F2) provides better blocking probability than $\mathrm{F}(\mathrm{w}, \mathrm{l})$ with the traditional shortest-route-based selection (denoted by SP). The performance gap between $\mathrm{F}(\mathrm{w}, \mathrm{l})$ with $\mathrm{F} 1$ (or F2) and $\mathrm{F}(\mathrm{w}, \mathrm{l})$ with SP with non-uniform traffic distribution is wider than that with uniform traffic distribution. The configuration in Fig. 5 (c) has two $s-d$ pairs with unusually high traffic intensity (making it more unbalanced in some sense). The performance gap is wider in Fig. 5 (c) than Fig 5 (b). This is intuitively pleasing - the performance improvement due to route selection with global perspective (i.e. F1 and F2) is more for more unbalanced call traffic.

The simulation results presented so far indicates that among our three algorithms, the $\mathrm{F}(\mathrm{w}, \mathrm{l})$ with predetermined routes computed by F2 has the best performance. LLR can be improved by employing offline route selection method F1 or F2, and which is better depends upon the configuration and environment. Between SPREAD and LLR, which performs better depends upon the configurations and environments (e.g., LLR is the winner in many of the NSFNET simulations while SPREAD completely outperforms LLR in a $5 \times 5$ mesh torus topology). Figs. 6-9 compare the performance of three algorithms $\mathrm{F}(\mathrm{w}, \mathrm{l})$ with F2, SPREAD, and LLR with F2.
(In all configurations presented in Figs 6-9, we have verified that $F 2$ yields the better performance than $F 1$ or the traditional shortest-path based approach both in $\mathrm{F}(\mathrm{w}, \mathrm{l})$ and LLR.) Fig 6 indicates that $\mathrm{F}(\mathrm{w}, \mathrm{l})$ performs the best among the three RWA algorithms for a sufficiently large number of fibers per link or for sufficiently large number of wavelengths ( $F \geq 8$ or $W \geq 8$ ). In many cases $\mathrm{F}($ w,l) performs significantly better than the other two. Incidentally, Fig. 6 shows that SPREAD performs better than LLR in some cases (e.g., $(W, F)=(8,8))$, and LLR performs better than SPREAD in other cases (e.g., $(W, F)=$ $(8,32),(W, F)=(8,64),(W, F)=(64,8),(W, F)=(48,48))$.

For non-uniform traffic in Figure 7 , we once again chose one $s-d$ pair and set its traffic intensity 10 times as high as other $s-d$ pairs. Nodes 1 and 2 in the NSFNET topology illustrated in Figure 2 were used as the $s-d$ pair that is heavily loaded. We can see that when $(W, F)=(48,4)$ and also when $(W, F)=(64,8), \quad \mathrm{F}(\mathrm{w}, \mathrm{l}) \quad$ significantly outperforms both SPREAD and LLR.

Fig. 8 shows the performance of the best three algorithms ( $\mathrm{F}(\mathrm{w}, \mathrm{l})-\mathrm{F} 2$, LLR-F2, and SPREAD) for an NSFNET topology having different numbers of fibers at different links. Fig. 8 indicates that $\mathrm{F}(\mathrm{w}, \mathrm{l})$ outperforms both Spread and LLR. Thus, Fig. 8 demonstrates the efficiency of the $F(w, l)$ algorithm for heterogeneous numbers of fibers at links.

Fig. 9 shows the simulation results of the $\mathrm{F}(\mathrm{w}, \mathrm{l})$, LLR, and SPREAD in the $5 \times 5$ mesh-torus topology. We note that for the simulations in Fig. 9, F(w,l) and LLR employed four predetermined routes per $s-d$ pair. In this regular topology, Fig. 9 indicates that $\mathrm{F}(\mathrm{w}, \mathrm{l})$ and SPREAD performs much better than LLR. At $(W, F)=(8,8), \mathrm{F}(\mathrm{w}, \mathrm{l})$ does not perform as well as SPREAD. However, as $F$ or $W$ increases, the performance of $\mathrm{F}(\mathrm{w}, \mathrm{l})$ and SPREAD becomes more or less the same. Fig. 9 also indicates that $\mathrm{F}(\mathrm{w}, \mathrm{l})$ outperforms SPREAD for sufficiently large values of $W$ and $F$. 


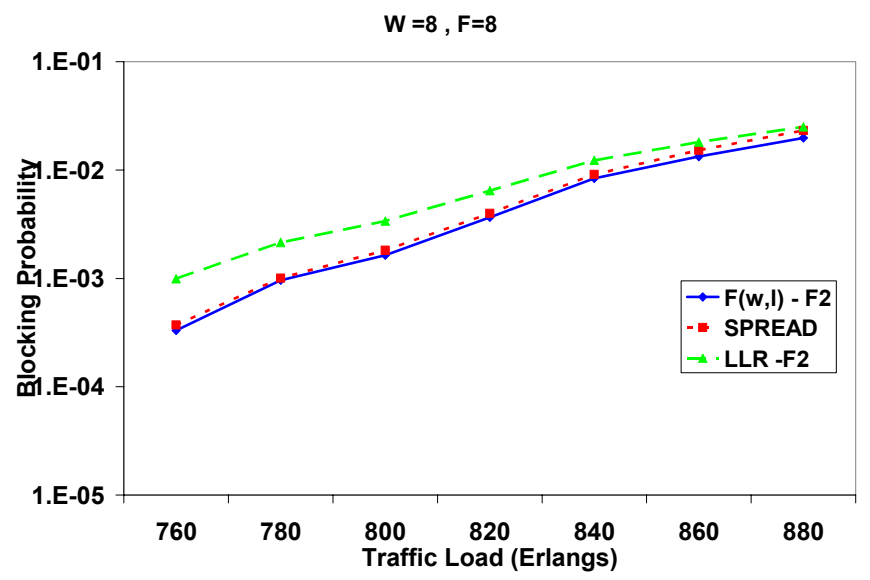

(a)

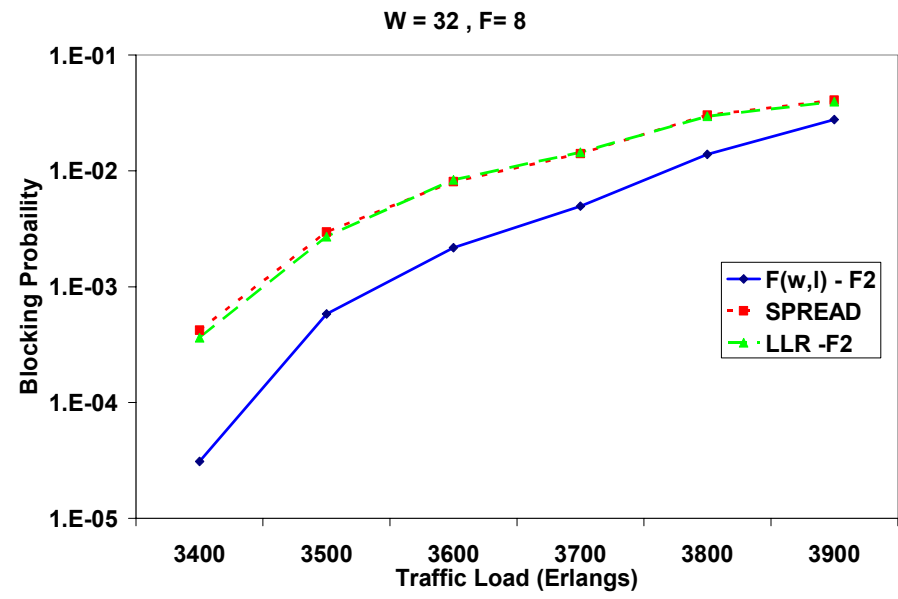

(c)

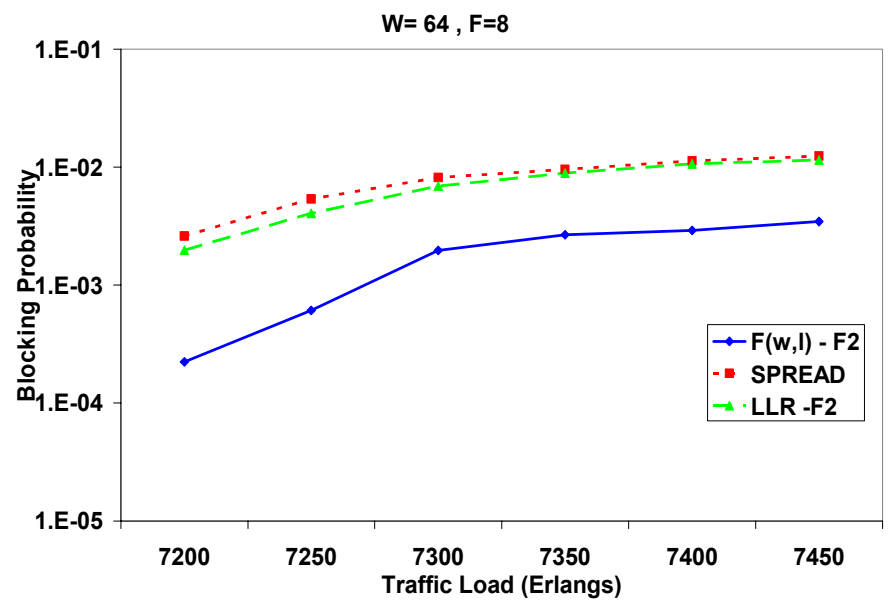

(e)

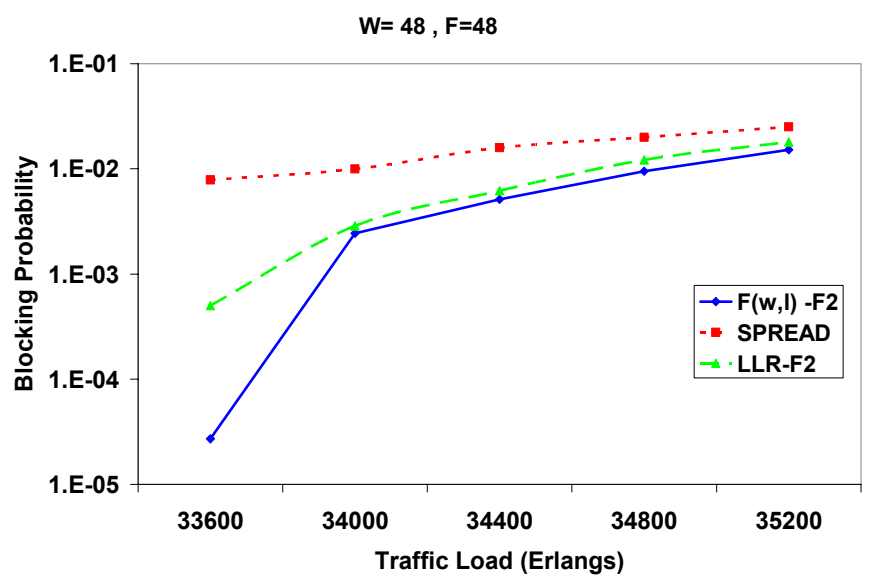

(b)

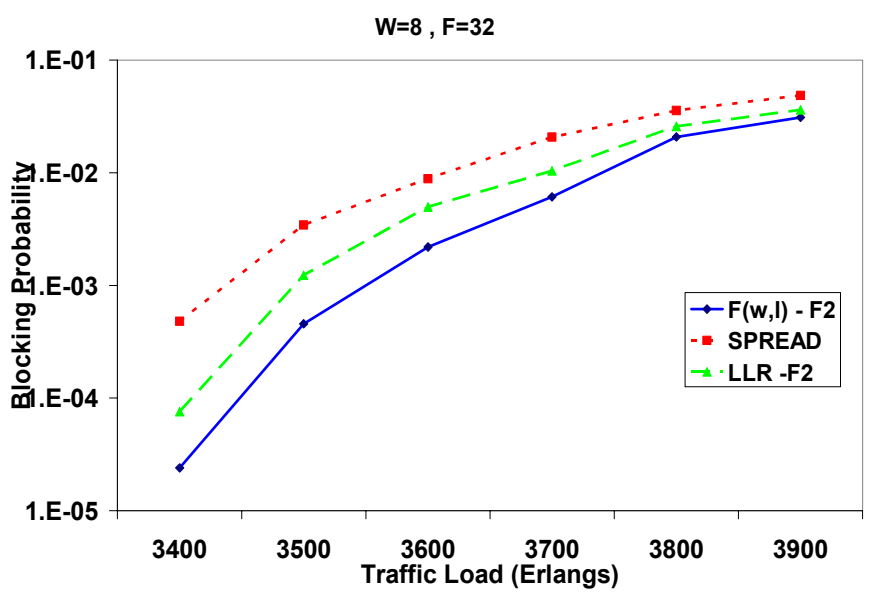

(d)

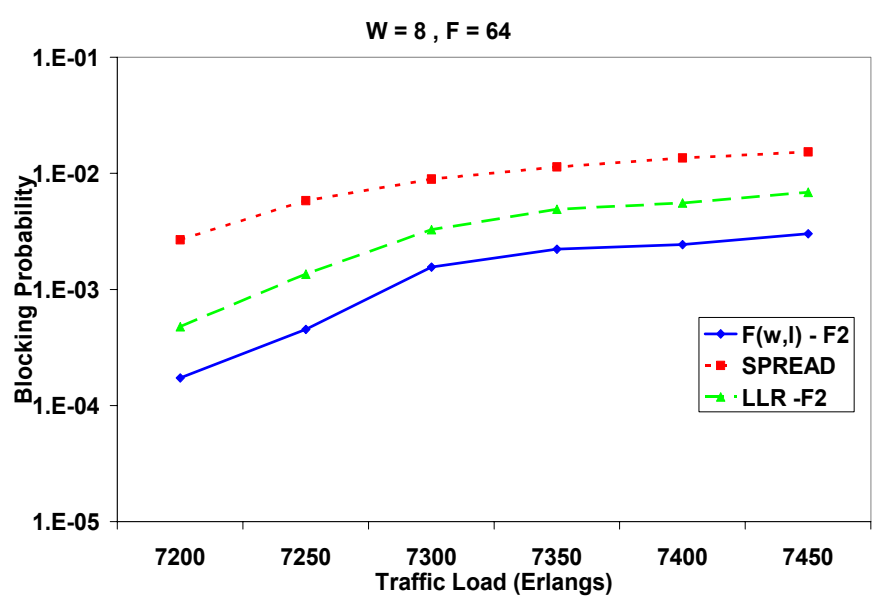

(f)

Fig. 6. Blocking probability vs. traffic load in NSFNET (Uniform Traffic Distribution) 


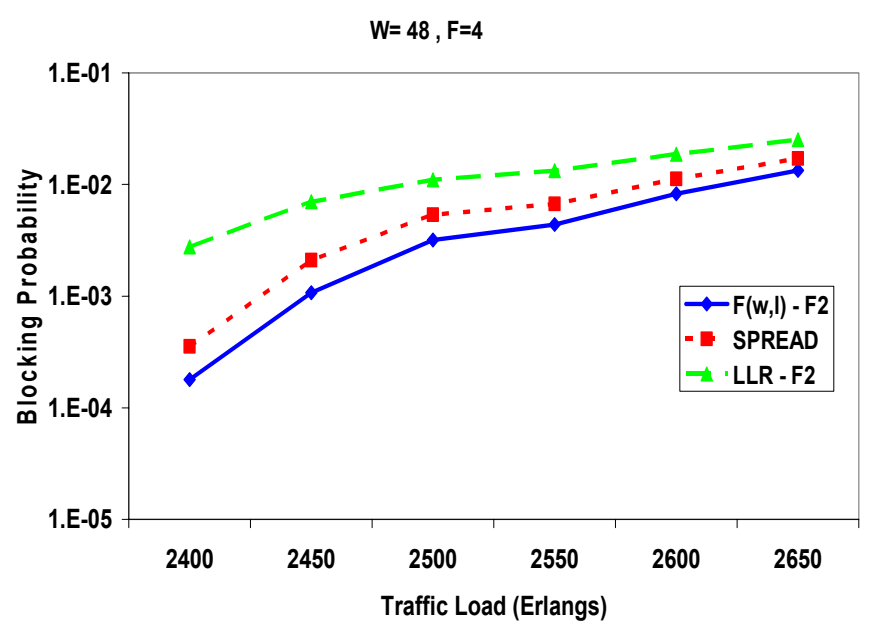

(a)

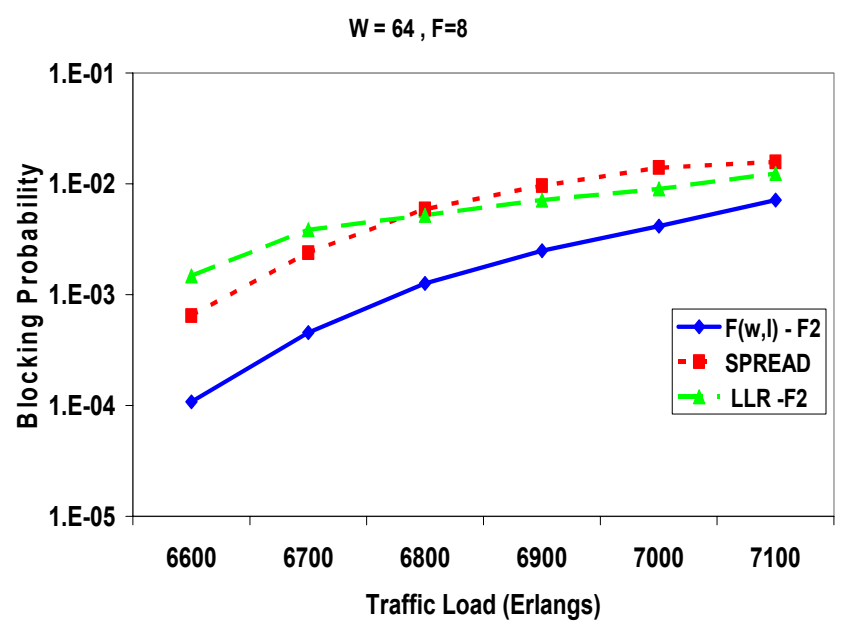

(b)

Fig. 7. Blocking probability vs. traffic load in NSFNET (non-uniform traffic)

a) $W=32, F=8,12$.

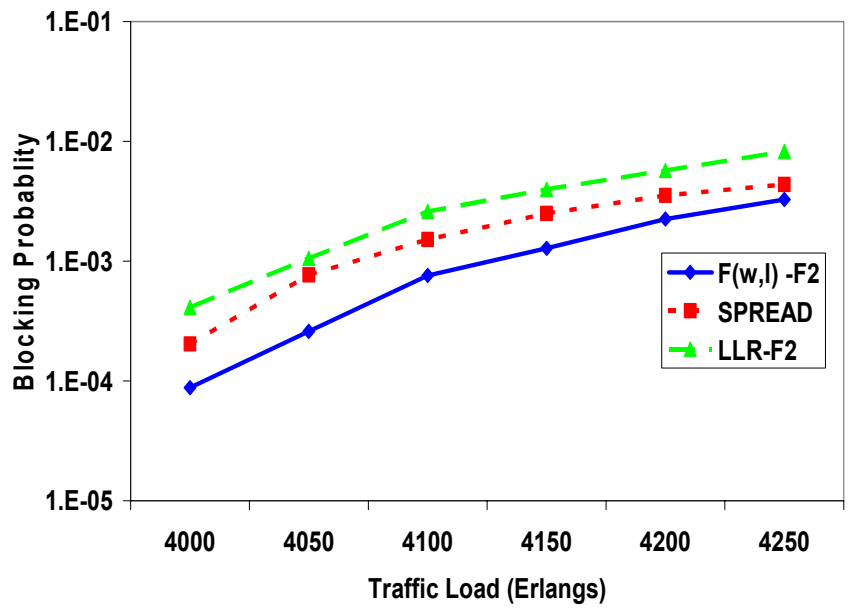

b) $W=64, F=8,12,16$.

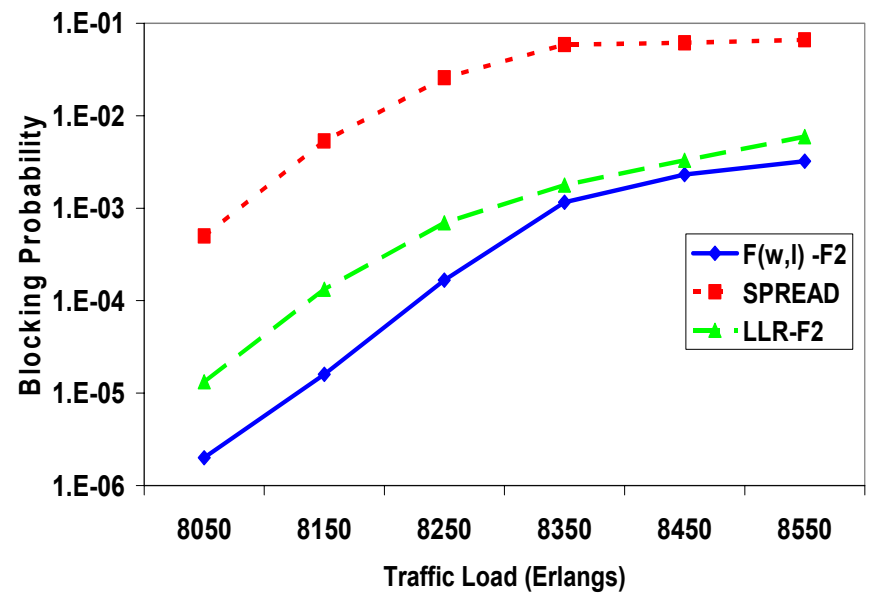

Fig. 8. Blocking probabilities in NSFNET in a heterogeneous number of fibers per link. (Uniform traffic distribution) 


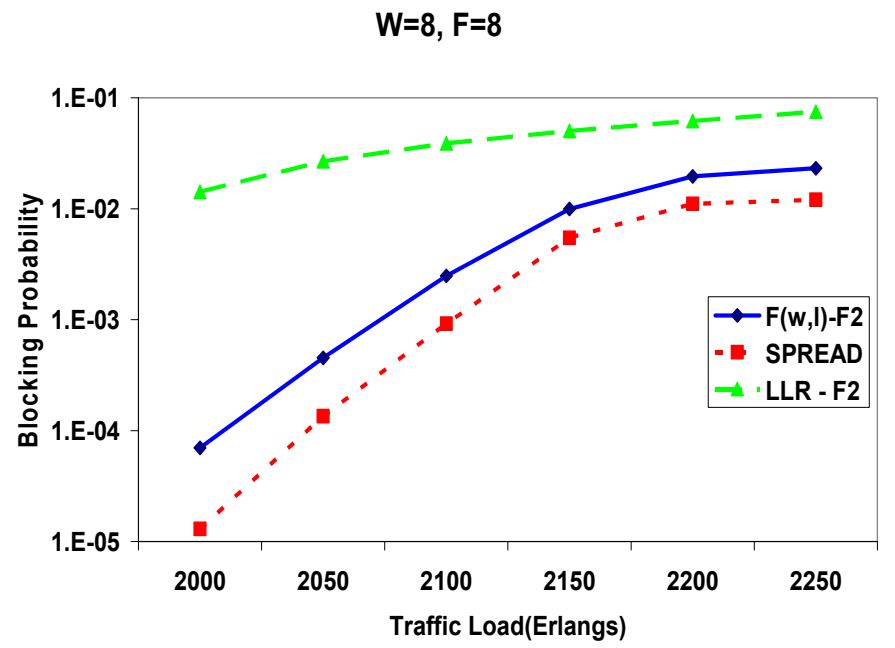

(a)

$W=32, F=8$

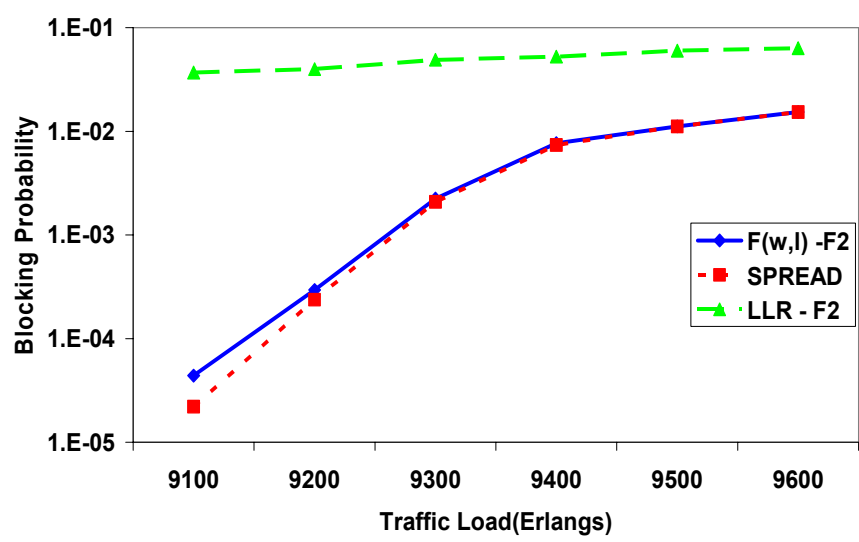

(b)

$W=64, F=8$

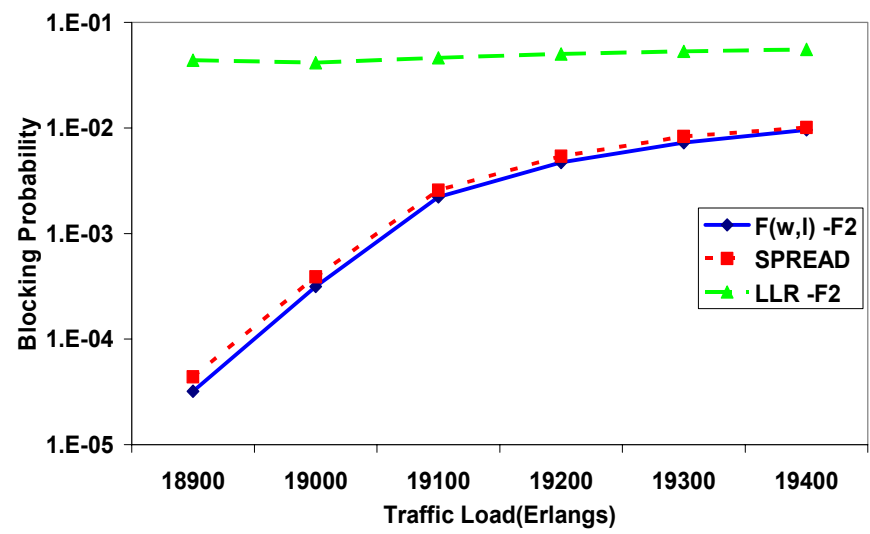

(d)
$W=8, F=32$

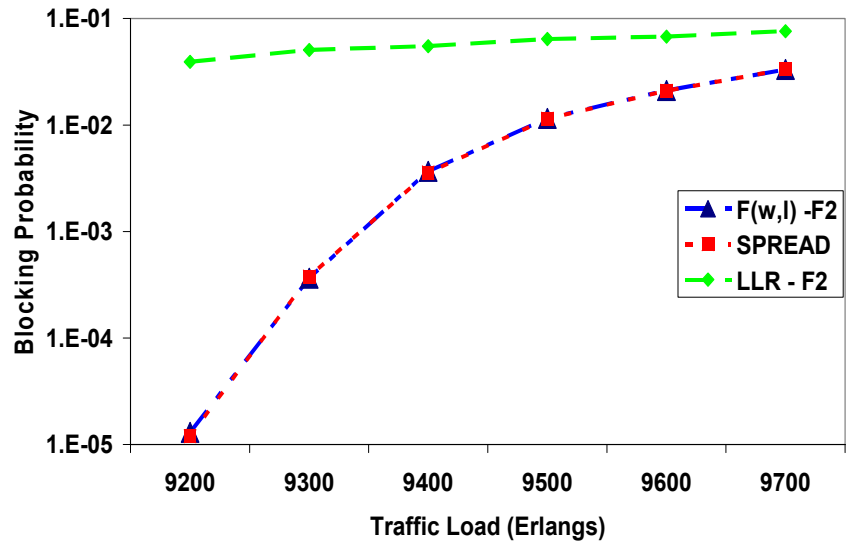

(c)

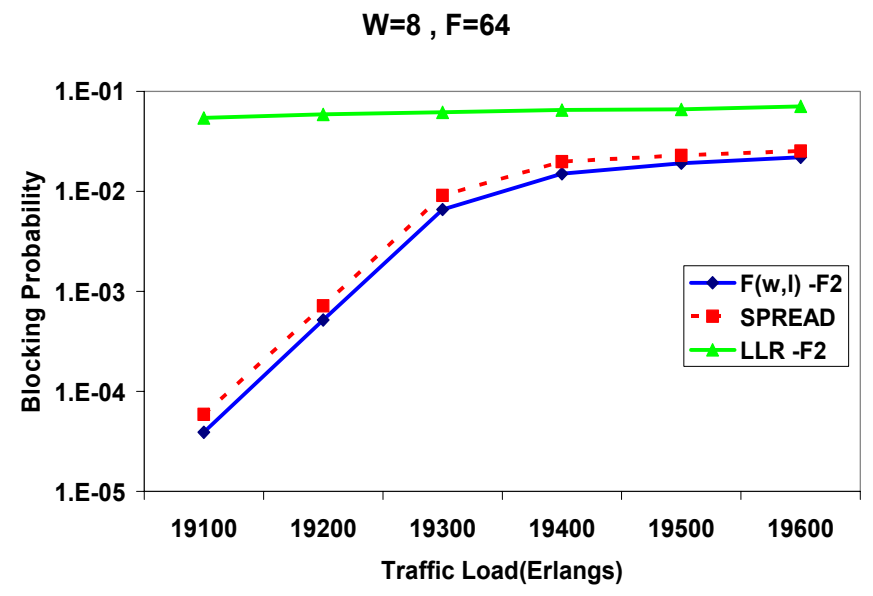

(e)

Fig 9. Blocking probability vs. traffic load in $5 \times 5$ mesh torus topology (Uniform traffic distribution) 


\section{SUMmaRY AND CONCLUSION}

We presented three dynamic routing and wavelength assignment algorithms - named $\mathrm{F}(\mathrm{w}, \mathrm{l}), \mathrm{MCR}$, and LSNLR based on some per-route variables - that are intended for multifiber WDM networks without wavelength converters. For networks with a large number of wavelengths per fiber and/or a large number of fibers per link, our algorithms exhibited particularly good performance. Therefore, our proposed RWA algorithms will be quite suitable for continuing advances in WDM technology. In particular, algorithm $\mathrm{F}(\mathrm{w}, \mathrm{l})$ significantly outperforms other existing algorithms in terms of blocking probabilities. Also, $\mathrm{F}(\mathrm{w}, \mathrm{l})$ requires much less online computation for call processing than well-known algorithms such as SPREAD.

The three algorithms presented in this paper make a routing decision on the basis of per-route status and subsequently choose a wavelength. Thus, this paper illustrates that in designing a heuristic RWA algorithm for multifiber networks with a large number of wavelengths per fiber and/or a large number of fibers per link, a separation between routing and wavelength assignment problems with an appropriate perroute metric to select the desired route can have positive effects on reducing the blocking probability.

This paper also illustrates that the choice of predetermined routes can significantly affect the blocking probability of an RWA. As a method of selecting the set of predetermined routes for RWAs based on predetermined routes we suggested integer linear programming formulations that can balance the traffic load in the network. We tested these methods of selecting predetermined routes for a few RWAs that are based on predetermined routes, including $\mathrm{F}(\mathrm{w}, \mathrm{l})$ and LLR. Our simulation results show that the proposed methods result in significantly lower blocking probabilities than the traditional shortest-route-based method.

\section{REFERENCES}

[1] B.Mukherjee, Optical Communication Networks. New York: McGrawHill, 1997.

[2] J. P. Jue, Design and Analysis of Architecture and Protocols for WDM Optical Networks. Ph.D. Dissertation, University of California, Davis, 1999.

[3] G. Jeong and E. Ayanoglu, "Comparison of wavelength-interchanging and wavelength-selective cross-connects in multiwavelength all-optical networks," Proc. IEEE INFOCOM'96, pp. 156-163.

[4] S. Baroni, P. Bayvel, R. J. Gibbens, and S. K. Korotky, "Analysis and design of resilient multifiber wavelength-routed optical transport," IEEE Journal of Lightwave Technology, vol 17, May 1999, pp. 743-758

[5] S. Xu, L. Li and S. Wang, "Dynamic routing and assignment of wavelength algorithms in multifiber wavelength division multiplexing networks," IEEE J. Select Areas Commun., vol. 18, no. 10, Oct. 2000, pp. 2130-2137.

[6] A. Mokhtar and M. Azizoglu, "Adaptive wavelength routing in all-optical networks," IEEE/ACM Trans. Networking. vol. 6, Apr. 1998, pp. 197-206.

[7] K. C. Lee and V. O. K. Li, "A wavelength convertible optical network," J. of Lightwave Tech., vol 11, no. 5/6, May/June 1993, pp. 962-970.

[8] S. Subramaniam, M. Azizoglu, and A. Somani, "All-optical networks with sparse wavelength conversion," IEEE/ACM Trans. Networking, vol. 4, no. 4, August 1996, pp. 544-557.

[9] R. Ramaswami and G. Sasaki, "Multiwavelength optical networks with limited wavelength conversion," Proc. IEEE INFOCOM'97, pp. 489-498.
[10] B. Ramamurthy and B. Mukherjee, "Wavelength conversion in WDM networking," IEEE J. Selec. Areas in Commun., vol 16, Sep. 1998, pp. 1061-1073.

[11] V. Sharma and E. A. Varvarigos, "Limited wavelength translation in alloptical WDM mesh networks," Proc. IEEE INFOCOM'98, vol. 2, 1998, pp. 893-901.

[12] H. Zang, J. P. Jue, and B. Mukherjee, "A review of routing and wavelength assignment approaches for wavelength-routed optical WDM networks," SPIE/Baltzer Optical Networks Magazine (ONM), vol. 1, no. 1, Jan. 2000, pp. 47-60.

[13] I. Chlamtac, A. Faragó, and T. Zhang, "Lightpath (wavelength) routing in large WDM networks," IEEE J. Select Areas Commun., vol. 14, no. 5, June 1996, pp. 909-913.

[14] K. Chan and T. P. Yum, "Analysis of least congested path routing in WDM lightwave networks," Proc. IEEE INFOCOM'94, vol. 2, 1994, pp. 962-969.

[15] L. Li and A. K. Somani, "Dynamic wavelength routing using congestion and neighborhood information," IEEE/ACM Trans. Networking, vol. 7, no. 5, Oct. 1999, pp. 779-786.

[16] E. Karasan and E. Ayanoglu, "Effect of wavelength routing and selection algorithm on wavelength conversion gain in WDM optical networks," IEEE/ACM Trans. Networking, vol. 6, Apr. 1998, pp. 186-196.

Jong-Seon Kim received the B.S. and M.S. degrees in electronics engineering from Korea University, Seoul, Korea, in 1988 and 2000, respectively. He is working towards the $\mathrm{Ph} . \mathrm{D}$. degree in the Department of Electrical Engineering, University of Southern California, CA, USA. From 2004, he has been also a Principal Engineer in the Samsung Electronics Co., Ltd., Yongin, Korea. His research interests include optical WDM networks, routing algorithms, and performance evaluation.

Daniel C. Lee (S'91-M'92) received the Ph.D. (1992) and M.S. (1987) degrees from the Massachusetts Institute of Technology in electrical engineering \& computer science. He received a B.S. (1985) degree in electrical engineering with honors and a B.S. (1985) degree in mathematics from the University of Maryland at College Park. From 1993 to 1998, he devoted his research to the systems engineering of networks and communication systems at the U.S. Naval Research Laboratory (NRL) in Washington, DC. At the Center for Computational Science in NRL, he participated in the development of an object oriented protocol software framework, CASiNO. At the Naval Space Center in NRL, Dr. Lee developed a proxy agent for managing the ICEbox network, a U.S. government information-dissemination system. In 1998, Dr. Lee joined the faculty of Electrical Engineering Department at the University of Southern California, where his main research interests have been quality of service and resource allocation issues in communication systems and networks. Applications of his research include wireless communications and networking, sensor networks, optical networks, and internet multimedia. He is currently an Associate Professor in the School of Engineering Science at Simon Fraser University, Burnaby, BC, Canada.

Dr. Lee's honors include the Alan Berman Research Publication Award from NRL in 1995, the U.S. Navy's Outstanding Performance Award at NRL in 1995, and the Frederick C. Hennie III Teaching Award from MIT in 1989. He recently coauthored a book, Quality of Service for Internet Multimedia, Upper Saddle River, NJ: Prentice Hall, 2004c.

Harsha Sridhar received the Eng. degree in electronics and instrumentation engineering from Visveswariah Technological University, India, in 2003. Currently, he is a graduate student at the Department of Computer Science and Engineering at Wright State University, Dayton, Ohio, USA. His research interests include computer and communication networks, optical networks and wireless communications. 